\title{
Regularización laboral de trabajadores de la salud pagados con recursos del Seguro Popular en México
}

\author{
Gustavo Nigenda, PhD, ${ }^{(1,2)}$ José Arturo Ruiz-Larios, L en Soc, ${ }^{(2)}$ \\ Matilde Elizabeth Aguilar-Martínez, L en Com, ${ }^{(2)}$ Rosa Bejarano-Arias, $L$ en Soc.(2)
}

\begin{abstract}
Nigenda G, Ruiz-Larios JA, Aguilar-Martínez ME, Bejarano-Arias R. Regularización laboral de trabajadores de la salud pagados con recursos del Seguro Popular en México. Salud Publica Mex 2012;54:616-623.
\end{abstract}

\begin{abstract}
Resumen
Objetivo. Describir y analizar el proceso de regularización del personal de salud pagado por el Sistema de Protección Social en Salud de México. Material y métodos. Se utilizan datos primarios y secundarios provenientes de la evaluación del Sistema de Protección Social en Salud en 2009. Resultados. La regularización mejora las condiciones laborales de los trabajadores pero sus implicaciones para el conjunto del sistema no son necesariamente positivas. Conclusiones. Se requiere considerar la necesidad de que este tipo de inversiones beneficien a todos los actores interesados, principalmente la población asegurada por el sistema.
\end{abstract}

Palabras clave: recursos humanos en salud; condiciones de trabajo; política social
Nigenda G, Ruiz-Larios JA,

Aguilar-Martínez ME, Bejarano-Arias R.

Labor regularization of health workers paid with funds from the Mexican System for Social Protection in Health. Salud Publica Mex 2012;54:616-623.

\begin{abstract}
Objective. The process of regularization of workers paid by the Social Protection Health System of Mexico is described and analyzed. Materials and methods. Primary and secondary data collected by the external evaluation of the Mexican System for Social Protection in Health in 2009 were used. Results. The regularization clearly improved the labor conditions of workers contracted by the system but a broader systemic implication of regularization does not seem to be necessarily positive. Conclusion. It is important to consider the need to guarantee that this type of changes in the contractual conditions of workers benefit all actors, particularly the insured population.
\end{abstract}

Key words: Health manpower; working conditions; public policy
L os sistemas de salud del mundo continúan enfrentando la disyuntiva de cómo integrar de manera adecuada al personal de salud para que participe, activamente y de manera motivada, en la producción de servicios de salud dirigidos a las poblaciones beneficiarias. En este fenómeno, tanto la formación como la incorporación laboral de los trabajadores de la salud juegan un papel importante. ${ }^{1}$

Por un lado, los trabajadores de la salud deben pasar por largos periodos de entrenamiento a fin de garantizar que sean técnicamente capaces de llevar a cabo las tareas que las instituciones les asignan. Por

(I) Harvard Global Equity Initiative. EUA.

(2) Instituto Nacional de Salud Pública. Cuernavaca, México.

Fecha de recibido: 14 de octubre de 20II • Fecha de aceptado: 8 de junio de 2012

Autor de correspondencia: Matilde Elizabeth Aguilar Martínez. Av. Periférico Sur 4809,

Col. El Arenal Tepepan. I46I0 México, DF.

Correo electrónico: elizabeth.aguilar@insp.mx 
otro, los trabajadores de la salud en el mundo son mayoritariamente asalariados de las instituciones públicas de salud lo cual, en cierta medida, los ha hecho vulnerables a los ajustes macroeconómicos de los regímenes laborales, tanto en países industrializados como en vías de desarrollo. ${ }^{2}$

Diversos estudios han mostrado que los trabajadores de la salud han sido expuestos a procesos de flexibilización laboral.* Siguiendo recomendaciones de organismos internacionales vinculados al sistema financiero mundial, muchos países en desarrollo, a partir de los años ochenta, ajustaron sus modelos económicos para reducir, en algunos casos, y para reorientar, en algunos otros, el gasto público, así como incrementar la participación del sector privado en áreas productivas y de servicios, y mantener el control sobre la fuerza de trabajo y los mercados laborales. ${ }^{3}$

La flexibilización laboral se caracteriza principalmente por la inestabilidad en el empleo, un férreo control sobre los salarios (lo cual reduce en el tiempo su poder adquisitivo) y la restricción de beneficios tales como jubilación, primas vacacionales, días de descanso, entre otras prestaciones sociales y económicas. Las experiencias más comunes de contratación reportadas en países desarrollados se han visto marcadas por este contexto. ${ }^{4}$

Otro efecto importante en el campo laboral ha sido la reducción de la capacidad de los sindicatos para representar los intereses de los trabajadores, tanto permanentes como de aquellos que se vinculan a través de contratos temporales. Se ha observado también una disminución del índice de sindicalización, ${ }^{\neq, 5}$ la cual está asociada con el avance de la flexibilización laboral.

Por otra parte, los sistemas de salud han tenido que responder en los últimos 20 años a nuevas presiones generadas por el cambio demográfico, caracterizado principalmente por el envejecimiento y por los consecuentes cambios epidemiológicos caracterizados principalmente por el aumento de las enfermedades crónico degenerativas y su coexistencia con las enfermedades transmisibles. ${ }^{6}$

Para enfrentar estos cambios, diversos países de América Latina iniciaron procesos de reforma, los cuales han derivado en un aumento en el gasto en salud, frecuentemente a expensas del gasto público, que se ha visto reflejado en aumentos en infraestructura,

\footnotetext{
* Algunos autores prefieren utilizar el concepto de precarización para denotar el deterioro de las condiciones laborales de los trabajadores a partir de las políticas de ajuste estructural de los años ochenta.

* El índice de sindicalización es la proporción del total de los trabajadores asalariados que está afiliada a un sindicato (OIT, 2003).
}

equipamiento y modernización tecnológica, pero no necesariamente en mejores condiciones laborales para los trabajadores de la salud.

En México, el Sistema de Protección Social en Salud (SPSS), creado en 2003 a partir de una modificación a la Ley General de Salud, transfiere recursos financieros a los estados para garantizar la prestación de servicios a poblaciones no cubiertas por la seguridad social. ElSPSS ha intentado imprimir una lógica de financiamiento por subsidios a la demanda para sustituir la lógica de los presupuestos históricos a fin de fortalecer la capacidad de elección de la población. ${ }^{7}$

Desde su diseño original, los fondos del Seguro Popular (brazo operativo del SPSS) estaban diseñados para financiar el costo directo de la producción de los servicios de salud y una parte importante de estos fondos se ha dedicado a contratar personal de salud, inicialmente bajo una modalidad que no incluía ningún tipo de protección laboral. A partir de 2008 la modalidad de contratación varió, extendiendo el tiempo y costo del contrato y ofreciendo algunos beneficios a los trabajadores, tales como el goce de vacaciones, aguinaldo, servicio médico y días económicos. Este proceso es conocido como regularización.

Para llevar a cabo la regularización, el sistema de salud mexicano enfrenta una disyuntiva importante entre contratar al personal manteniendo las restricciones laborales prevalentes en el sector público hasta la fecha, o generar nuevas condiciones reconociendo los derechos laborales de los trabajadores, bajo el supuesto de que su participación podrá apoyar la obtención de objetivos institucionales. El presente documento propone que para entender el fenómeno de la vinculación de los recursos humanos a los sistemas de salud, éste debe plantearse tanto desde una postura laborista interesada en la mejora de las condiciones de trabajo, como desde una perspectiva sistémica que busque una vinculación acorde con las capacidades financieras del sistema y la participación efectiva de los trabajadores en el logro de los objetivos.

El presente artículo analiza algunas de las implicaciones de la decisión de regularizar a miles de trabajadores de la salud en México con los recursos de un programa que busca asegurar el financiamiento de la atención a la salud de millones de familias mexicanas que no cuentan con cobertura de seguridad social.

\section{Material y métodos}

Los datos que se presentan en el artículo fueron obtenidos de la Evaluación del Sistema de Protección Social en Salud del año 2009, proyecto financiado por la Dirección General de Evaluación del Desempeño de la Secretaría de 
Salud y autorizado por el Comité de Ética del Instituto Nacional de Salud Pública. Se llevó a cabo en nueve entidades federativas (Baja California, Campeche, Distrito Federal, Guerrero, Hidalgo, Jalisco, Morelos, Querétaro y Zacatecas) seleccionadas a partir de los siguientes criterios: a) forma jurídica del Régimen Estatal de Protección Social en Salud (REPSS); b) desempeño en evaluaciones previas; c) ubicación geográfica; d) tiempo de incorporación al SPSS; y d) contratación público-privada para la prestación de servicios. ${ }^{8}$

El estudio tuvo un diseño mixto de técnicas cuantitativas y cualitativas. Se recolectaron datos específicos sobre la contratación de recursos humanos con financiamiento del SPSS en los años 2008 y 2009 (número, perfil, modalidad de contratación -regularizados y honorarios-, sueldos por categorías y modalidades de contratación), a partir de los registros estatales de los Servicios de Salud (SESA), los REPSS y de la Comisión Nacional de Protección Social en Salud (CNPSS).

La información cualitativa se obtuvo a través de 36 entrevistas semiestructuradas realizadas con personal directivo y mandos medios del SPSS y la Secretaría de Salud, de los ámbitos estatal y federal. Las entrevistas abordaron dos ejes temáticos: la contratación del personal con recursos del SPSS y el proceso de regularización. Todas las entrevistas fueron grabadas en audio, previo consentimiento informado de los entrevistados.

También se llevó a cabo la revisión de diversos documentos oficiales relacionados con el marco legal para la contratación del personal y el funcionamiento del SPSS, tales como la Ley General de Salud, los Reglamentos de la Ley General de Salud en materia de Protección Social en Salud y de la Comisión Nacional de Protección Social en Salud, la Ley Federal de los Trabajadores al Servicio del Estado, la Ley del Instituto de Seguridad y Servicios Sociales de los Trabajadores del Estado, las Condiciones Generales de Trabajo suscritas entre la Secretaría de Salud y el Sindicato Nacional de los Trabajadores de la Secretaría de Salud (SNTSA), el Catálogo de Puestos y Tabulador de Sueldos de las Ramas Médica, Paramédica y grupos afines del año 2007, el acuerdo de creación de la Comisión Nacional para la Reglamentación de la Contratación de Recursos Humanos en las Entidades Federativas (CNRCRHEF) y su manual de funcionamiento; así como el anexo IV del Acuerdo de Coordinación para la ejecución del SPSS. Los datos cuantitativos fueron sistematizados y procesados en una base de datos creada en excel, mientras que para la información cualitativa se construyeron matrices en word por tipo de informante y entidad federativa.

Para el análisis de la información cualitativa se llevó a cabo la triangulación de fuentes, a fin de corroborar y complementar la información obtenida, mientras que los datos cuantitativos fueron analizados a través de estadística descriptiva, figuras y correlaciones.

\section{Resultados}

\section{Antecedentes de la regularización}

Desde el inicio del proceso de ajuste estructural en México, el volumen de trabajadores con plaza de base se ha mantenido prácticamente constante hasta la fecha en la mayoría de instituciones públicas y la modalidad de contratación dominante ha sido el contrato temporal sin beneficios de ley. El gobierno federal no ha creado nuevas plazas de base en ninguna de las categorías laborales. ${ }^{9}$ La Secretaría de Salud (SSa), tanto a nivel federal como estatal, empleó 284142 trabajadores en $2008,{ }^{10}$ la mayoría de ellos con plaza de base.

Un elemento clave para entender la política de contratación de la SSa en los últimos 30 años es el proceso de descentralización de los servicios de salud. Como parte de este proceso se diseñaron un conjunto de políticas federales en recursos humanos, que definan la nueva relación entre la SSa y los trabajadores. El impacto de la política de ajuste en los salarios y la restricción para la creación de nuevas plazas es conocida $^{11}$ pero políticamente el SNTSA logró resistir la presión para descentralizarse y así mantener capacidad de negociación en el ámbito federal. De esta manera, los trabajadores de base pagados por los estados fueron homologados en sus condiciones laborales en relación con las condiciones que tenían los trabajadores pagados por la nómina federal. ${ }^{12}$

El único antecedente documentado de creación de plazas es el de los trabajadores eventuales contratados por el Programa de Ampliación de Cobertura (PAC) a finales de los años noventa. El PAC empleó a miles de trabajadores para apoyar las acciones de salud en las zonas de más difícil acceso, cuyos salarios fueron cubiertos inicialmente por préstamos del Banco Internacional de Reconstrucción y Fomento, con el compromiso de que la responsabilidad de pagar los salarios en las fases subsecuentes del programa fuera asumida por la SSa. A inicios del año 2000 el PAC operaba con 5417 personas ${ }^{13}$ y fue en ese mismo año que el SNTSA y la SSa negociaron el otorgamiento de plazas de base para la mayoría del personal operativo del programa. Esta fue la única ocasión desde 1992 que la Secretaría de Salud generó plazas nuevas.

\section{Negociaciones para la regularización}

Una proporción importante de los fondos enviados por la Comisión Nacional de Protección Social en Salud a los estados se ha destinado a la contratación de 
diferentes categorías de personal. En los primeros años predominó la contratación por honorarios, ${ }^{*}$ con duración de cinco meses y medio, generalmente sin acceso a las prestaciones laborales establecidas en la legislación correspondiente. ${ }^{14}$

En julio de 2006 se creó la Comisión Nacional para la Reglamentación de la Contratación de Recursos Humanos en las Entidades Federativas (CNRCRHEF) y se empezó a plantear la regularización de los trabajadores en situación precaria. Precario ${ }^{\ddagger}, 3$ fue el término que algunos actores políticos utilizaron para señalar a los trabajadores bajo contrato temporal, el cual se volvió discurso común en todos los ámbitos de la SSa.

Uno de los factores que desencadenó la regularización fue la reforma a la Ley del Instituto de Seguridad y Servicios Sociales de los Trabajadores del Estado, publicada en marzo de 2007, donde se plantea la integración del personal contratado por honorarios a los beneficios establecidos en dicha ley. El SNTSA fue uno de los actores que tomó la iniciativa de negociar un cambio en las condiciones de contratación de los trabajadores pagados con fondos del SPSS. Este gremio participó activamente para la creación de CNRCRHEF, donde lograron convocar la participación del Comisionado Nacional del SPSS, del Secretario de Salud federal y sus representantes administrativos, y de altas autoridades de la Secretaría de Hacienda y Crédito Público (SHCP). El incremento de fondos asignado para la operación del SPSS, en medio de un alza en el precio internacional del petróleo en 2006, generó una atmósfera de posibilidad sobre un aumento en el gasto público, no vista en años anteriores. ${ }^{16}$

Un acuerdo inicial dentro de la CNRCRHEF fue que la duración de los contratos sería extendida de cinco a doce meses con renovación automática. Los salarios serían actualizados con base en el tabulador correspondiente y se incluirían beneficios tales como seguridad social, aguinaldo, jubilación, pensión, vacaciones, prima vacacional, aunque en menor cantidad y proporción a las que marca la Ley Federal de los Trabajadores al Servicio del Estado y más limitadas en comparación con las contenidas en las Condiciones Generales de Trabajo (la modalidad burocrática de un contrato colec-

\footnotetext{
* Régimen fiscal de prestación de servicios profesionales, en el cual las personas que prestan sus servicios de manera independiente establecen un tipo de relación laboral sumamente laxa. Se trata más de una relación mercantil que laboral con la entidad a la que prestan sus servicios.

* La situación precaria se caracteriza por la corta duración del contrato, el alto riesgo de despido, lo flexible del marco legal, las bajas remuneraciones y el carecer de organización colectiva, entre otros aspectos poco favorables para los trabajadores.
}

tivo de trabajo). ${ }^{8}$ Además, el SNTSA tendría derecho a recibir el pago de la cuota sindical por cada trabajador regularizado así como participar en la selección de los individuos a "regularizar". La CNPSS pagaría con sus fondos los salarios líquidos y la SHCP generaría una partida especial para financiar los beneficios sociales y prestaciones. No hubo definición en términos de la modalidad de contratación para los nuevos trabajadores que fueran contratados posteriormente al acuerdo.

El SNTSA cuenta con más de 200000 afiliados y 101 secciones sindicales distribuidas en las 32 entidades federativas e instituciones dependientes de la SSa. El SNTSA es herencia del modelo corporativista del Estado prevaleciente en México. ${ }^{7}$ Este sindicato se ha distinguido por su postura oficialista, negociadora, poco beligerante, aunque también ha enarbolado demandas y espacios propios frente a políticas como la descentralización de los servicios de salud, por ejemplo. En el proceso de regularización el SNTSA ha formado parte activa e influyente en la CNRCRHEF, así como en las subcomisiones estatales creadas como parte del mismo proceso.

Un elemento clave para entender las consecuencias de este fenómeno es que desde 2004 la CNPSS se percató de la autonomía de los estados para ejercer el gasto repercutía en que los recursos se siguieran utilizando bajo la lógica de subsidio a la oferta y no como subsidio a la demanda, además de que se usaran para financiar bienes y servicios sin una lógica de eficiencia. Esto se demostró en algunos estados que llegaron a utilizar 70\% de los recursos recibidos en compras de medicamentos o en contratación de personal. ${ }^{14}$ Para el año 2007, la CNPSS promovió que en el anexo IV del Acuerdo de Coordinación que se firma entre la CNPSS y los estados para la ejecución de los recursos, se establecieran límites de gasto. Para medicamentos se fijó "hasta 30\%" y para la contratación de personal el límite se definió "hasta 40\%".

\section{Avances de la regularización}

La puesta en práctica del acuerdo entre la CNPSS y la SHCP muestra diferencias importantes en relación con sus contenidos originales. Una divergencia es que la SHCP no ha generado una nueva partida para financiar los beneficios asociados con la regularización. El costo total es cubierto con los fondos transferidos por la CNPSS a los estados, lo cual ha implicado un conjunto de problemas en el proceso. En principio, por solicitud de la CNPSS en la CNRCRHEF, en todo el país se limitó a la regularización de médicos y enfermeras, por ser considerado personal directamente relacionado con las intervenciones del CAUSES, lo cual generó descontento 


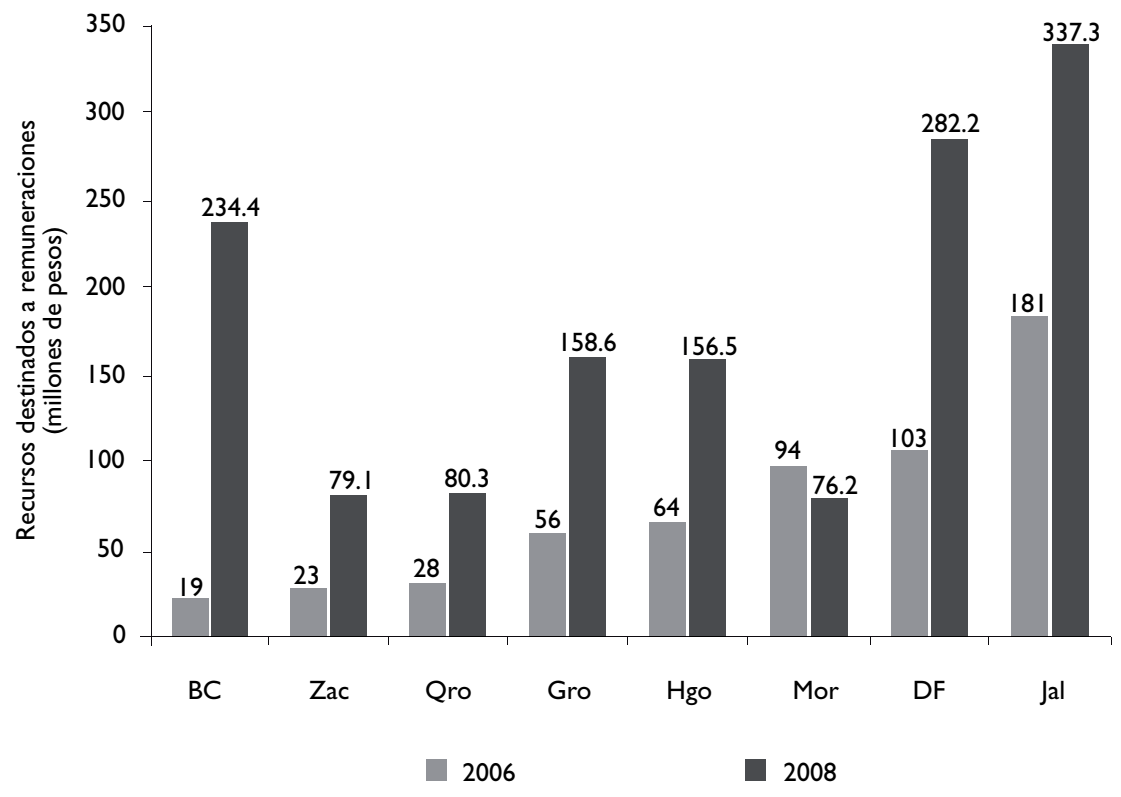

Fuente: Referencia 8

Figura I. Recursos destinados a Remuneración del personal contratado con fondos del SPSS en ocho estados. Méxıco, 2006-2008

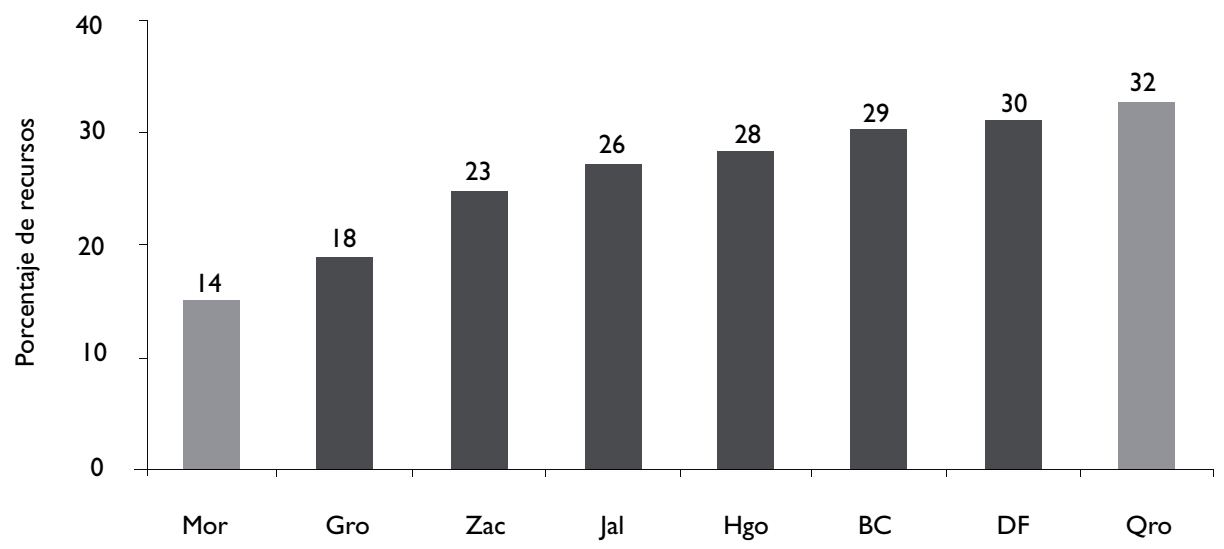

Fuente: Referencia 8

Figura 2. Porcentaje de recursos destinados a remuneración del personal contratado con fondos del SPSS en ocho estados. México, 2008

en términos de desempeño de los sistemas estatales de salud no son completamente evidentes. Sin embargo, a través de las entrevistas con funcionarios es posible plantear algunas de ellas de manera inicial.

Un elemento importante a señalar es que la mayor parte de los trabajadores regularizados creen que su nueva condición es equivalente a la de un trabajador con plaza de base debido a que ahora pagan cuotas sindicales. Bajo ese estatus, los trabajadores tienden a pensar que su pensión está garantizada al cumplir la edad estipulada por ley, ya que el riesgo de que sean despedidos es prácticamente nulo. También saben que 
la posibilidad del despido no depende de su desempeño cotidiano y que el sindicato tendrá capacidad para interceder ante la institución, en caso necesario.

Sin embargo, la condición de regularizado no es equivalente a una plaza de base. Si en la productividad así como en la capacidad técnica y el trato a los usuarios muestran un desempeño debajo del promedio, pueden comprometer la renovación del contrato. Por otra parte, son pocos los SESA que cuentan con mecanismos para llevar a cabo la supervisión del desempeño de los trabajadores.

Una consecuencia inmediata que ha sido expuesta en el apartado anterior es que el impacto financiero de la regularización ha sido absorbido en su totalidad por los fondos del SPSS asignados a cada estado. A ello hay que sumar las opiniones de funcionarios del sistema, en el sentido de que el desempeño de los trabajadores se modificará negativamente con el nuevo estatus, e incluso algunos señalan que los cambios empiezan ya a expresarse mediante faltas injustificadas, retardos y actitudes negativas en relación con el trabajo y con los usuarios de los servicios. Es necesario explorar este tipo de consecuencias en los próximos años.

\section{Discusión}

El cambio de estatus laboral de 24534 trabajadores de la salud, pagados con fondos del SPSS en 2009, fue producto de una negociación entre instituciones públicas y el SNTSA. Los términos de la negociación fueron eminentemente políticos, sin una definición clara de las bases técnicas y financieras. Los actores beneficiados son el personal que obtuvo incrementos salariales y beneficios sociales, además del SNTSA que logró erigirse como representante de estos trabajadores y recibir las cuotas sindicales correspondientes.

Las implicaciones de este fenómeno para el desempeño de los sistemas locales de salud son enormes. A partir de la inexistencia de un acuerdo formal por escrito sobre el proceso y los alcances de la "regularización", con reglamentaciones específicas, los fundamentos de la negociación se expresan en los hechos de manera discrecional y todas las partes buscan obtener beneficios. Los SESA y los REPSS tienen el doble compromiso de utilizar fondos para pagar a los trabajadores y sus nuevas condiciones, pero también requieren restringir el gasto a $40 \%$ del total de fondos disponibles mensualmente (con base en el anexo IV del Acuerdo de Coordinación). Uno de los ajustes que algunos estados tuvieron que hacer en 2009 fue reducir el número de trabajadores contratados para la prestación de servicios.

Las nuevas condiciones de contratación de los trabajadores regularizados mejoran su situación laboral en un ambiente de fuertes presiones para las finanzas públicas del país, exacerbadas a partir de la crisis declarada en agosto de 2008. A pesar de que no se trata de un proceso que generó nuevas plazas de base, la regularización de este grupo de trabajadores es un hecho inédito en los últimos 25 años en México, porque propone una forma novedosa de vincular a los trabajadores con el sistema. La coyuntura financiera y política permitió que el acuerdo se estableciera y se iniciara su ejecución. La disponibilidad de fondos en el SPSS fue clave para tomar la decisión, la cual además fue ejecutada por actores institucionales federales y estatales. Sin embargo, esto muestra que la lógica operativa y financiera del programa no fue considerada en la decisión, ponderando los intereses de actores individuales por encima del SPSS y sus beneficiarios.

Esta experiencia es valiosa para otros programas que pretenden incorporar personal de salud para lograr sus metas sanitarias. Si bien, es fundamental que se busque una mejoría en las modalidades de contratación de los trabajadores, el planteamiento de un proceso de este tipo debe ser financiera y técnicamente planeado a fin de generar resultados positivos para todos los actores involucrados, pero principalmente para los programas y las poblaciones que se benefician de ellos.

La limitación más importante del presente estudio es la escasa información que se obtuvo para analizar otros efectos de la contratación del personal regularizado, particularmente en relación con su desempeño. Si bien esta información fue obtenida a través de entrevistas con gerentes del sistema, en el futuro es importante llevar a cabo una medición rigurosa de su desempeño en términos de eficiencia y calidad a fin de obtener una visión más clara de los dividendos generados por la inversión realizada en su contratación.

Finalmente, no es posible plantear que la regularización representa un cambio en la política de contratación de la SSA ni del gobierno federal, la cual mantiene criterios de flexibilización laboral. Como se mostró, la coyuntura para este proceso favoreció un desenlace que permitió mejorar ciertas condiciones de trabajo pero que implicó la erogación de fondos no previstos por parte del SPSS para cubrir salarios y beneficios, y el incumplimiento hasta la fecha de la parte del acuerdo correspondiente a la SHCP. El siguiente paso es saber si estas son las condiciones adecuadas para fomentar el desempeño de los trabajadores contratados bajo esta modalidad.

\section{Agradecimientos}

Al maestro Edson Serván Mori por su apoyo en algunos de los cálculos presentados. La evaluación de procesos administrativos del SPSS en 2009, incluido su compo- 
nente de recursos humanos, fue contratada por la Dirección General de Evaluación del Desempeño, a quien se agradece este apoyo. Sin embargo, los contenidos expresados en el artículo son responsabilidad absoluta de sus autores.

Declaración de conflicto de intereses: Los autores declararon no tener conflicto de intereses.

\section{Referencias}

I. Organización Mundial de la Salud. Potenciar al máximo el personal sanitario existente. En Informe sobre la salud en el mundo 2006. Colaboremos por la salud. Ginebra: OMS, 2006.

2. Simoens $S$, Hurst J. The supply of physician services in OECD countries. OECD Health working papers No. 2I. Paris: OECD, 2006.

3. Dal Poz M, Galin P, Novick M, Varella T. Relaciones laborales en el sector salud. Fuentes de información y métodos de análisis. Quito: OPS, 2000. 4. International Labour Office. Terms of employment and working conditions in health sector reforms. Report for discussion at the Joint Meeting on Terms of Employment and Working Conditions in Health Sector Reforms. Ginebra: ILO, 1998.

5. Comisión para la Cooperación Laboral. Nota informativa. Cambios recientes en los índices de sindicalización en América del Norte [monografía en Internet]. Washington: Comisión para la Cooperación Laboral, 2003. [Consultado 20II septiembre] Disponible en: http://www.naalc. org/spanish/pdf/april_03_spanish.pdf

6. Organización Mundial de la Salud. Informe sobre la salud en el mundo 2000. Mejorar el desempeño de los sistemas de salud. Ginebra: OMS, 2000 7. Nigenda G. El seguro popular de salud en México. Desarrollo y retos para el futuro. Nota técnica de salud. No.2/2005. Washington: Banco Interamericano de Desarrollo, 2005.
8. Nigenda G, González-Robledo LM, Aracena B, Juárez C, Wirtz V, Idrovo AJ, et al. Evaluación de procesos administrativos del Sistema de Protección Social en Salud 2009. Resumen ejecutivo. México: INSP, 2010. 9. Ruiz JA, Molina J, Nigenda G. Médicos y mercado de trabajo en México. En: Knaul F, Nigenda G, ed. El Caleidoscopio de la salud. México: Funsalud, 2003.

10. Secretaría de Salud, Dirección General de Información en Salud. Boletín de Información Estadística. México: SSa, 2008.

II. López-Arellano O, Blanco-Gil J. La polarización de la política de salud en México. Cad Saúde Pública 2001;17(I):43-54.

12. Nigenda G, Ruiz JA. El caso de México. En: Organización Panamericana de la Salud, Organización Mundial de la Salud. Factores restrictivos para la descentralización en recursos humanos. Programa de Desarrollo de Recursos Humanos. División de Desarrollo de Sistemas y Servicios de Salud. Serie Desarrollo de Recursos Humanos No. 16. Washington: OPS, 1999.

13. Secretaría de Salud. Programa de Ampliación de Cobertura 19962000. Recuento y testimonio de un esfuerzo de equidad y extensión de servicios de salud en México, SSa, 2000.

I4. Nigenda G, Aguilar E, Ruiz JA, Bejarano R. Recursos Humanos. En: Sistema de Protección Social en Salud. Evaluación de procesos administrativos. México: INSP, 2008.

15. Gerry y Janine Rodgers (coord.) Les emplois precaires dans la regulation du marche du travail. La croissance du travail atypique en Europe de I'Ouest. ILO, Ginebra, 1990.

16. Scott J. Seguro Popular incidence analysis. En: World Bank. Decentralized service delivery for the poor. México: World Bank, 2006.

17. Nigenda G, Solórzano A. Doctors and corporatist politics: The case of the Mexican medical profession. J Health Polit Policy Law, 1997;22:73-99. 